\title{
Anisotropic plasmon-coupling dimerization of a pair of spherical electron gases
}

\author{
Godfrey Gumbs ${ }^{1,2}$, Andrii Iurov ${ }^{1}$, Antonios Balassis, ${ }^{3}$ Danhong Huang ${ }^{4}$ \\ ${ }^{1}$ Department of Physics and Astronomy, Hunter College of the \\ City University of New York, 695 Park Avenue, New York, NY 10065, USA \\ 2 Donostia International Physics Center (DIPC), \\ $P$ de Manuel Lardizabal, 4, 20018 San Sebastian, Basque Country, Spain \\ ${ }^{3}$ Physics Department, Fordham University 441 East Fordham Road, Bronx, NY 10458, USA \\ ${ }^{4}$ Air Force Research Laboratory, Space Vehicles Directorate Kirtland Air Force Base, NM 87117, USA
}

(Dated: May 9, 2022)

\begin{abstract}
We have discovered a novel feature in the plasmon excitations for a pair of Coulomb-coupled nonconcentric spherical two-dimensional electron gases (S2DEGs). Our results show that the plasmon excitations for such pairs depend on the orientation with respect to the external electromagnetic probe field. The origin of this anisotropy of the inter-sphere Coulomb interaction is due to the directional asymmetry of the electrostatic coupling of electrons in excited states which depend on both the angular momentum quantum number $L$ and its projection $M$ on the axis of quantization taken as the probe E-field direction. We demonstrate the anisotropic inter-sphere Coulomb coupling in space and present semi-analytic results in the random-phase approximation both perpendicular and parallel to the axis of quantization. For the incidence of light with a finite orbital or spin angular momentum, the magnetic field generated from an induced oscillating electric dipole on one sphere can couple to an induced magnetic dipole on another sphere in a way depending on the direction parallel or perpendicular to the probe $\mathbf{E}$ field. Such an effect from the plasmon spatial correlation is expected to be experimentally observable by employing circularly-polarized light or a helical light beam for incidence. The S2DEG serves as a simple model for fullerenes as well as metallic dimers, when the energy bands are far apart.
\end{abstract}

PACS numbers: 73.20.-r， 73.20.Mf，78.20.Bh，78.67.Bf

\section{INTRODUCTION}

Recent calculations on the plasma excitations of a spherical two-dimensional electron gas (S2DEG) have yielded some interesting behaviors as functions of the angular momentum quantum number $L$ and the radius $R$ of the shell [1 3]. In those model calculations, the electron gas is assumed to be confined to an infinitesimally thin shell which is embedded in a medium with background dielectric constant $\epsilon_{b}$. The plasma excitation frequencies were shown not to depend on the projection $M$ of angular momentum $L$ on the axis of quantization. This degeneracy is expected due to retained rotational symmetry and there is no energy dispersion which arises in the case for the cylindrical nanotube [4, 5]. Interest in plasmon excitations in fullerenes dates back to the work by Östling, et al. [6] who used a spherical shell model to examine the experimental data for plasma resonances in $\mathrm{C}_{60}[$ [7, 8]. The model for the plasmons described in Ref. [6] assumes that the buckyball is doped and its active modes are attributed to either dipole or monopole-like excitations. Since our system is neutral, only dipole-like plasmon modes will exist.

The interest in the S2DEG has been generated by the observation that fullerenes $9-15]$ span an entire family from "buckybabies" with thirty-two carbon atoms and radius $0.35 \mathrm{~nm}$ to very large fullerenes with four thousand, eight hundred and sixty atoms and radius $3.141 \mathrm{~nm}$ [ 13]. These molecules can be modified into other molecular configurations which make them very versatile. It is such an adaptability that gives them enormous practical applications in materials science, electronics and nanotechnology.

The model we employ, which consists of a S2DEG confined to the surface, allows us to investigate the electronic properties, related to their spherical shape and the lattice structure [16 [18], and to neglect their radial motion [19] at the same time. These electronic properties include the collective plasma excitations, electron energy loss spectra as well as the thermoelectric properties of fullerenes. This is conceptually similar to the electron gas model for a carbon nanotube, which has been studied extensively [4, 20, 21]. The only variables in our calculations are the radius, the separation between two displayed shells and the number of free electrons on each shell. The plasma formula contains these parameters, as well as the orbital angular momentum, and we vary them to examine how the plasmon frequency depends on them.

The authors of Ref. 22] attempted to obtain the plasma excitations for a pair of displaced S2DEGs whose centers do not coincide. However, the formalism by Rotkin and Suris 22 is incomplete since these authors did not include the full Coulomb coupling between the two S2DEGs in their Eqs. (3) and (4). Consequently, the Eq. (8) in their paper for plasma excitations has missed the Coulomb matrix elements coupling all the possible angular momenta on the two 
spheres, meaning that we cannot use the angular momentum to label plasma excitations, or $L$ is no longer a good quantum number for the system considered, as they have done in their Eq. (9). Another interesting subtlety which arises from the Coulomb dimer is worth commenting on at this point. Suppose we chose the axis of quantization for angular momentum to be along the $z$ direction. Let us assume that one of the S2DEGs has its center at the origin as shown in Fig.2 We then have a choice of placing the second S2DEG with its center on the $z$-axis or on the $x$-axis. From a mathematical point of view, the Coulomb matrix elements involved have values which depend on the spatial coordinates. From a physical point of view, once the axis of quantization is chosen, the spherical symmetry in the Coulomb dimer is broken, resulting in a dependence of location for the second S2DEG. Similar calculations of the polarization functions proved the existence of strongly localized image states near the surface of a buckyball [23].

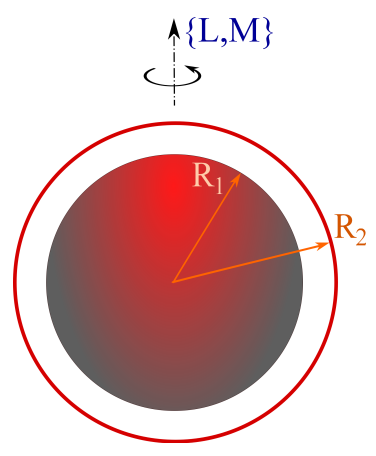

FIG. 1: (Color online) Schematic illustration of a pair of concentric shells with inner radius $R_{1}$ and outer radius $R_{2}$. Here, $L$ and $M$ are angular momentum quantum number and component for spheres.

In this paper, we are particularly interested in calculating the plasma excitations of two Coulomb coupled S2DEGs. These may model either a double shell onion-type buckyball as illustrated in Fig. 1 or a pair of non-overlapping buckyballs whose centers have a finite separation between them (called Coulomb dimer), as depicted in Fig.2. Each S2DEG may be polarized by external electromagnetic fields. However, the S2DEG is only polarized for finite angular momentum quantum number $L \neq 0$. Additionally, $L$ is still a good quantum number for labeling the plasma excitations on concentric shells. Here, for simplicity, $L=0$ corresponds to a non-circularly-polarized probe field, while $L=1$ is associated with a circularly-polarized probe field. Moreover, the higher angular momentum with $L>1$ can be achieved by a special light beam, e.g., a helical light beam. However, when two S2DEGs have their centers well separated so that there is no overlap of their charge distributions, the breaking of the spherical symmetry leads to significant differences with the double-wall buckyball, as we now describe.

When the two shells are concentric, the Coulomb interaction between them enters the plasmon mode equation through its dependence on the angular momentum quantum number $L$ as well as the radius of each shell. This type of Coulomb coupling only leads to a renormalization of each of the two plasmon modes which exist on each shell independent of the S2DEG on the other. The resulting coupled modes are in-phase symmetric and out-of-phase antisymmetric charge-density oscillations. However, as far as the Coulomb dimer is considered, the polarization functions for all values of $L$ on each sphere are coupled to each other. The inter-sphere Coulomb matrix element depends on both $L$ and its projection $M$ on the axis of quantization. Therefore, in principle, the plasmon mode equation is given in terms of a determinant of infinite dimension. But, the corresponding matrix may be divided into diagonal sub-matrices corresponding to $L=1,2,3, \cdots$ and consisting of $2(2 L+1) \times 2(2 L+1)$ elements whose Coulomb interactions depend on $L$ and $(2 L+1)$ values of $M$ for each of the two shells. The off-diagonal sub-matrices, on the other hand, involve Coulomb matrix elements which depend on pairs of different angular momenta, $L$ and $L^{\prime}$, arising from each sphere. These off-diagonal Coulomb terms are generally smaller than their diagonal counterparts and so may be formally treated as perturbations. Consequently, in the lowest-order approximation, the $L=1$ mode is split by Coulomb interactions depending on $M=0, \pm 1$ on each sphere, leading to the occurrence of three symmetric and three antisymmetric plasmon modes. Additionally, we derive semi-analytic expressions for these Coulomb matrix elements for large separations. The Coulomb interaction described by the diagonal sub-matrices directly lead to the spatial correlation between plasmons on two spheres (or simply called the Coulomb dimerization for short).

In Sec.II] we will first formulate the method for calculating the plasmon equations on a pair of non-overlapping S2DEGs. This is based on the random-phase approximation (RPA) in evaluating the induced density fluctuations for a weak external perturbation. Section [II is devoted to a discussion of our numerical results. Some concluding remarks are given in Sec.IV] Mathematical details of our calculations are provided in Appendices. 


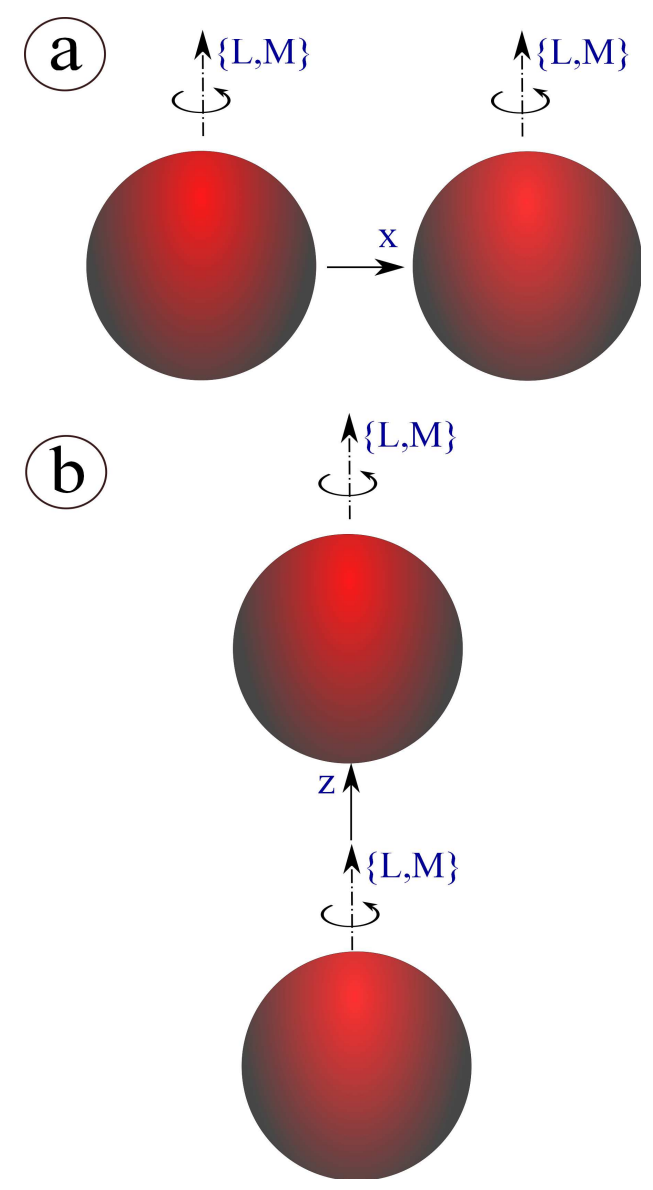

FIG. 2: (Color online) Schematic illustration of a pair of displaced buckyballs. The axis of quantization is along the $z$ direction with angular momentum quantum number $L$ and component $M$. In (a), one buckyball has its center at the origin and the other is centered on the $x$-axis. In (b), one buckyball has its center at the origin while the other is centered on the $z$-axis.

\section{GENERAL FORMULATION OF THE PROBLEM}

\section{A. Plasmon-Mode Equation for a Double-Walled Nano-Sphere}

We first use linear-response theory to derive the plasma mode equation for a S2DEG on a pair of concentric shells with inner radius $R_{1}$ and outer radius $R_{2}$. If an electron is confined on the surface of a sphere of radius $R$, the eigenfunctions and eigenenergies are

$$
\begin{gathered}
<\mathbf{r} \mid \alpha>\equiv \psi_{\alpha}(r, \theta, \varphi)=\frac{\mathcal{R}(r)}{R} Y_{\ell, m}(\Omega), \quad \mathcal{R}^{2}(r)=\delta(r-R), \\
\varepsilon_{\alpha}=\frac{\ell(\ell+1) \hbar^{2}}{2 \mu^{*} R^{2}}
\end{gathered}
$$

with $R=R_{1}$ or $R_{2}, Y_{\ell, m}(\Omega) \equiv Y_{\ell, m}(\theta, \phi)$ being the spherical harmonic function, $\mu^{*}$ the effective mass of electrons, $\alpha=\{\ell, m\},|m| \leq \ell$ and $\ell=0,1,2, \cdots$. The density matrix is given by

$$
\begin{aligned}
& <\ell m\left|\hat{\rho}_{1}(\omega)\right| \ell^{\prime} m^{\prime}>=-2 e \sum_{i=1}^{2} \frac{f_{0}\left(\varepsilon_{\ell}^{i}\right)-f_{0}\left(\varepsilon_{\ell^{\prime}}^{i}\right)}{\hbar \omega+\varepsilon_{\ell}^{i}-\varepsilon_{\ell^{\prime}}^{i}} \\
& \times \sum_{L, M} \mathcal{F}_{L, M}\left(R_{i}, \omega\right) \int d \Omega Y_{\ell, m}^{*}(\Omega) Y_{L, M}(\Omega) Y_{\ell^{\prime}, m^{\prime}}(\Omega),
\end{aligned}
$$


where, after some calculation, we obtain from the Poisson equation with respect to the density fluctuation

$$
\frac{1}{r^{2}} \frac{d}{d r}\left[r^{2} \frac{d \mathcal{F}_{L, M}(r, \omega)}{d r}\right]-\frac{L(L+1)}{r^{2}} \mathcal{F}_{L, M}(r, \omega)=\sum_{i=1}^{2} \mathcal{A}_{L, M}^{(i)}(\omega) \delta\left(r-R_{i}\right)
$$

with $\omega$ being the external-field frequency and

$$
\mathcal{A}_{L, M}^{(i)}(\omega)=-\mathcal{F}_{L, M}\left(R_{i}, \omega\right) \frac{e^{2}}{\epsilon_{s} R_{i}^{2}} \Pi_{L}^{(i)}(\omega)
$$

Here, $\epsilon_{s} \equiv 4 \pi \epsilon_{0} \epsilon_{b}, \epsilon_{b}$ is the uniform background dielectric constant, the polarization function is given by

$$
\Pi_{L}^{(i)}(\omega)=2 \sum_{\ell, \ell^{\prime}} \frac{f_{0}\left(\varepsilon_{\ell}^{i}\right)-f_{0}\left(\varepsilon_{\ell^{\prime}}^{i}\right)}{\hbar \omega+\varepsilon_{\ell}^{i}-\varepsilon_{\ell^{\prime}}^{i}}(2 \ell+1)\left(2 \ell^{\prime}+1\right)\left(\begin{array}{ccc}
\ell & \ell^{\prime} & L \\
0 & 0 & 0
\end{array}\right)^{2}
$$

in terms of the Wigner $3 \mathrm{j}$-symbol, the Fermi-Dirac distribution function $f_{0}\left(\varepsilon_{\ell}^{i}\right)$ and the eigenenergies $\varepsilon_{\ell}^{i}$ are obtained from Eq. (2) by replacing $R$ with $R_{i}$.

Now, $\mathcal{F}_{L, M}(r, \omega)$ is explicitly given by

$$
r \mathcal{F}_{L, M}(r, \omega)=\left\{\begin{array}{cc}
\bar{C}_{1} r^{L+1}, & r<R_{1} \\
\bar{C}_{3} r^{L+1}+\bar{C}_{4} r^{-L}, & R_{1} \leq r \leq R_{2} \\
\bar{C}_{2} r^{-L}, & r>R_{2}
\end{array}\right.
$$

where $\bar{C}_{1}, \bar{C}_{2}, \bar{C}_{3}$ and $\bar{C}_{4}$ are some constants to be determined from the continuity of $\mathcal{F}_{L, M}(r, \omega)$ at $r=R_{1}, R_{2}$ and the step-like change of $\frac{d\left[r \mathcal{F}_{L, M}(r, \omega)\right]}{d r}$ at $r=R_{1}, R_{2}$. From these boundary conditions, we get

$$
\left[\begin{array}{cccc}
R_{1}^{L+1} & 0 & -R_{1}^{L+1} & -R_{1}^{-L} \\
0 & -R_{2}^{-L} & R_{2}^{L+1} & R_{2}^{-L} \\
-(L+1) R_{1}^{L} & 0 & (L+1) R_{1}^{L} & -L R_{1}^{-(L+1)} \\
0 & -L R_{2}^{-(L+1)} & -(L+1) R_{2}^{L} & L R_{2}^{-(L+1)}
\end{array}\right]\left[\begin{array}{c}
\bar{C}_{1} \\
\bar{C}_{2} \\
\bar{C}_{3} \\
\bar{C}_{4}
\end{array}\right]=\left[\begin{array}{c}
0 \\
0 \\
R_{1} \mathcal{A}_{L, M}^{(1)} \\
R_{2} \mathcal{A}_{L, M}^{(2)}
\end{array}\right]
$$

which we have solved for $\bar{C}_{1}, \bar{C}_{2}, \bar{C}_{3}$ and $\bar{C}_{4}$ and obtained

$$
\begin{aligned}
& \bar{C}_{1}=-\frac{R_{1}^{1-L}}{2 L+1} A_{L, M}^{(1)}(\omega)-\frac{R_{2}^{1-L}}{2 L+1} A_{L, M}^{(2)}(\omega) \\
& \bar{C}_{2}=-\frac{R_{1}^{L+2}}{2 L+1} A_{L, M}^{(1)}(\omega)-\frac{R_{2}^{L+2}}{2 L+1} A_{L, M}^{(2)}(\omega) \\
& \bar{C}_{3}=-\frac{R_{2}^{1-L}}{2 L+1} A_{L, M}^{(2)}(\omega) \\
& \bar{C}_{4}=-\frac{R_{1}^{L+2}}{2 L+1} A_{L, M}^{(1)}(\omega) .
\end{aligned}
$$

Combining Eqs. (5) and (7) and making use of the results for $\bar{C}_{1}$ and $\bar{C}_{2}$ in Eq. (9), we obtain the following pair of simultaneous equations

$$
\left[\begin{array}{cc}
1-\frac{e^{2}}{\epsilon_{s}(2 L+1) R_{1}} \Pi_{L}^{(1)}(\omega) & -\frac{e^{2}}{\epsilon_{s}(2 L+1) R_{1}}\left(\frac{R_{1}}{R_{2}}\right)^{L+1} \Pi_{L}^{(2)}(\omega) \\
-\frac{e^{2}}{\epsilon_{s}(2 L+1) R_{2}}\left(\frac{R_{1}}{R_{2}}\right)^{L} \Pi_{L}^{(1)}(\omega) & 1-\frac{e^{2}}{\epsilon_{s}(2 L+1) R_{2}} \Pi_{L}^{(2)}(\omega)
\end{array}\right]\left[\begin{array}{l}
\mathcal{F}_{L, M}\left(R_{1}, \omega\right) \\
\mathcal{F}_{L, M}\left(R_{2}, \omega\right)
\end{array}\right]=0
$$


Equation (10) has non-trivial solutions for $\mathcal{F}_{L, M}\left(R_{1}, \omega\right)$ and $\mathcal{F}_{L, M}\left(R_{2}, \omega\right)$ only if the determinant of the coefficient matrix is zero, i.e.,

$$
\begin{aligned}
& 1-\frac{e^{2}}{\epsilon_{s}(2 L+1)}\left[\frac{\Pi_{L}^{(1)}(\omega)}{R_{1}}+\frac{\Pi_{L}^{(2)}(\omega)}{R_{2}}\right] \\
& +\left[\frac{e^{2}}{\epsilon_{s}(2 L+1)}\right]^{2}\left[1-\left(\frac{R_{1}}{R_{2}}\right)^{2 L+1}\right] \frac{\Pi_{L}^{(1)}(\omega) \Pi_{L}^{(2)}(\omega)}{R_{1} R_{2}}=0 .
\end{aligned}
$$

\section{B. Plasmon Modes for a Pair of Displaced Spherical Shells}

We now turn our attention to a system of two spherical shells with their centers on the $x$ axis. The center of one of the spheres is at $x=0$ with radius $R_{1}$ whereas the other sphere is centered at $x=a$ and its radius is $R_{2}$. We assume that the inequality $a>R_{1}+R_{2}$ is satisfied to ensure no overlapping of charge distributions. In the absence of electron tunneling between the shells, the wave function for an electron on the $j$-th shell $(j=1,2)$ is given by

$$
<\mathbf{r} \mid j \nu>\equiv \Psi_{j, \ell m}\left(\mathbf{r}-(j-1) a \hat{\mathbf{e}}_{x}\right)=\frac{\mathcal{R}\left(r_{j}^{\prime}\right)}{R_{j}} Y_{\ell, m}(\Omega)
$$

with $\nu=\{\ell, m\}$ and $\mathcal{R}^{2}\left(r_{j}^{\prime}\right)=\delta\left(r_{j}^{\prime}-R_{j}\right)$. The energy spectrum has the form of Eq. (2)

$$
\varepsilon_{j, \nu}=\frac{\ell(\ell+1) \hbar^{2}}{2 \mu^{*} R_{j}^{2}}
$$

The equation of motion for the density matrix operator is

$$
\imath \hbar \frac{\partial \hat{\varrho}}{\partial t}=[\hat{\mathcal{H}}, \hat{\varrho}]_{-}
$$

where $\hat{\mathcal{H}}=\hat{\mathcal{H}}_{0}-e \Phi$ is the Hamiltonian of the electron on the surface of the sphere, $\hat{\mathcal{H}}_{0}$ is the free-electron Hamiltonian and $\Phi$ is the induced potential. The potential $\Phi$ satisfies Poisson's equation

$$
\nabla^{2} \Phi(\mathbf{r}, \omega)=\frac{4 \pi e}{\epsilon_{s}} \delta n(\mathbf{r}, \omega)
$$

Additionally, $\delta n(\mathbf{r}, \omega)$ is the induced electron-density fluctuation. We employ linear response theory (see Appendix A) to calculate the induced density fluctuation as

$$
\delta n(\mathbf{r}, \omega)=\sum_{j, j \prime} \sum_{\nu, \nu^{\prime}}<\mathbf{r}|j \nu><j \nu| \varrho_{1}(\mathbf{r}, \omega)\left|j^{\prime} \nu^{\prime}><j^{\prime} \nu^{\prime}\right| \mathbf{r}>
$$

where

$$
<j \nu\left|\varrho_{1}(\mathbf{r}, \omega)\right| j^{\prime} \nu^{\prime}>=-2 e \frac{f_{0}\left(\varepsilon_{j, \nu}\right)-f_{0}\left(\varepsilon_{j^{\prime}, \nu^{\prime}}\right)}{\hbar \omega+\varepsilon_{j, \nu}-\varepsilon_{j^{\prime}, \nu^{\prime}}}<j \nu|\Phi(\mathbf{r}, \omega)| j^{\prime} \nu^{\prime}>
$$

and we express the induced potential as $\Phi(\mathbf{r}, \omega)=\frac{1}{\mathcal{V}} \sum_{\mathbf{q}^{\prime}} \Phi\left(\mathbf{q}^{\prime}, \omega\right) e^{i \mathbf{q}^{\prime} \cdot \mathbf{r}}$ with $\mathcal{V}$ being the system volume. Then, Eq. (16) becomes

$$
\begin{aligned}
\delta n(\mathbf{r}, \omega) & =-\frac{2 e}{\mathcal{V}} \sum_{j, j^{\prime}} \sum_{\nu, \nu^{\prime}} \frac{f_{0}\left(\varepsilon_{j, \nu}\right)-f_{0}\left(\varepsilon_{j^{\prime}, \nu^{\prime}}\right)}{\hbar \omega+\varepsilon_{j, \nu}-\varepsilon_{j^{\prime}, \nu^{\prime}}}<\mathbf{r}\left|j \nu><j^{\prime} \nu^{\prime}\right| \mathbf{r}> \\
& \times \sum_{\mathbf{q}^{\prime}} \Phi\left(\mathbf{q}^{\prime}, \omega\right)<j \nu\left|e^{i \mathbf{q}^{\prime} \cdot \mathbf{r}}\right| j^{\prime} \nu^{\prime}>,
\end{aligned}
$$


or by taking the Fourier transform with respect to $\mathbf{r}$

$$
\begin{aligned}
\delta n(\mathbf{q}, \omega) & =-\frac{2 e}{\mathcal{V}} \sum_{j, j^{\prime}} \sum_{\nu, \nu^{\prime}} \frac{f_{0}\left(\varepsilon_{j, \nu}\right)-f_{0}\left(\varepsilon_{j^{\prime}, \nu^{\prime}}\right)}{\hbar \omega+\varepsilon_{j, \nu}-\varepsilon_{j^{\prime}, \nu^{\prime}}}<j^{\prime} \nu^{\prime}\left|e^{-i \mathbf{q} \cdot \mathbf{r}}\right| j \nu> \\
& \times \sum_{\mathbf{q}^{\prime}} \Phi\left(\mathbf{q}^{\prime}, \omega\right)<j \nu\left|e^{i \mathbf{q}^{\prime} \cdot \mathbf{r}}\right| j^{\prime} \nu^{\prime}>
\end{aligned}
$$

The matrix elements $\left\langle j \nu\left|e^{i \mathbf{q} \cdot \mathbf{r}}\right| j^{\prime} \nu^{\prime}>\right.$ with wave functions $\left.<\mathbf{r}\right| j \nu>$ given by Eq. (12) can be calculated based on the expansion of a plane wave in spherical waves

$$
e^{i \mathbf{q} \cdot \mathbf{r}}=4 \pi \sum_{L, M} i^{L} j_{L}(q r) Y_{L, M}^{*}\left(\Omega_{\mathbf{q}}\right) Y_{L, M}(\Omega)
$$

where $\Omega_{\mathbf{q}}=\left\{\theta_{\mathbf{q}}, \phi_{\mathbf{q}}\right\}$ in the $\mathbf{q}$-space and $j_{\ell}(x)$ is a spherical Bessel function. The result is

$$
\begin{aligned}
& <j \nu\left|e^{i \mathbf{q} \cdot \mathbf{r}}\right| j^{\prime} \nu^{\prime}>=4 \pi \delta_{j j^{\prime}} e^{i(j-1) q_{x} a} \\
\times & \sum_{L, M} i^{L} j_{L}\left(q R_{j}\right) Y_{L, M}^{*}\left(\Omega_{\mathbf{q}}\right) \int d \Omega Y_{\ell, m}^{*}(\Omega) Y_{L, M}(\Omega) Y_{\ell^{\prime}, m^{\prime}}(\Omega) .
\end{aligned}
$$

Substituting Eq. (21) into Eq. (19), we obtain after some algebra

$$
\begin{aligned}
& \delta n(\mathbf{q}, \omega)=-(4 \pi)^{2} \frac{2 e}{\mathcal{V}} \sum_{\ell, m} \sum_{l^{\prime}, m^{\prime}} \sum_{j=1}^{2} \frac{f_{0}\left(\varepsilon_{j, \ell}\right)-f_{0}\left(\varepsilon_{j, \ell^{\prime}}\right)}{\hbar \omega+\varepsilon_{j, \ell}-\varepsilon_{j, \ell^{\prime}}} e^{-i(j-1) q_{x} a} \\
& \times \sum_{L, M}(-i)^{L} j_{L}\left(q R_{j}\right) Y_{L, M}\left(\Omega_{\mathbf{q}}\right) \int d \Omega Y_{\ell^{\prime}, m^{\prime}}^{*}(\Omega) Y_{L, M}^{*}(\Omega) Y_{\ell, m}(\Omega) \\
& \times \sum_{q_{x}^{\prime}, \mathbf{q}_{\perp}^{\prime}} e^{i(j-1) q_{x}^{\prime} a} \Phi\left(q_{x}^{\prime}, \mathbf{q}_{\perp}^{\prime}, \omega\right) \\
& \times \sum_{L^{\prime}, M^{\prime}} i^{L^{\prime}} j_{L^{\prime}}\left(q^{\prime} R_{j}\right) Y_{L^{\prime}, M^{\prime}}^{*}\left(\Omega_{\mathbf{q}^{\prime}}\right) \int d \Omega^{\prime} Y_{\ell, m}^{*}\left(\Omega^{\prime}\right) Y_{L^{\prime}, M^{\prime}}\left(\Omega^{\prime}\right) Y_{\ell^{\prime}, m^{\prime}}\left(\Omega^{\prime}\right),
\end{aligned}
$$

where $\mathbf{q}_{\perp}^{\prime}=\left\{q_{y}^{\prime}, q_{z}^{\prime}\right\}$, or

$$
\begin{aligned}
& \quad \delta n(\mathbf{q}, \omega)=-(4 \pi) \frac{2 e}{\mathcal{V}} \sum_{j=1}^{2} \sum_{L, M} \sum_{\ell, \ell^{\prime}} \frac{f_{0}\left(\varepsilon_{j, \ell}\right)-f_{0}\left(\varepsilon_{j, \ell^{\prime}}\right)}{\hbar \omega+\varepsilon_{j, \ell}-\varepsilon_{j, \ell^{\prime}}}(2 \ell+1)\left(2 \ell^{\prime}+1\right)\left(\begin{array}{ccc}
\ell & \ell^{\prime} & L \\
0 & 0 & 0
\end{array}\right)^{2} \\
& \times e^{-i(j-1) q_{x} a} j_{L}\left(q R_{j}\right) Y_{L, M}\left(\Omega_{\mathbf{q}}\right) \\
& \times \sum_{q_{x}^{\prime}, \mathbf{q}_{\perp}^{\prime}} e^{i(j-1) q_{x}^{\prime} a} \Phi\left(q_{x}^{\prime}, \mathbf{q}_{\perp}^{\prime}, \omega\right) j_{L}\left(q^{\prime} R_{j}\right) Y_{L, M}^{*}\left(\Omega_{\mathbf{q}^{\prime}}\right) .
\end{aligned}
$$

Taking the Fourier transform of Eq. (15), we have $\Phi(\mathbf{q}, \omega)=-\frac{4 \pi e}{\epsilon_{s} q^{2}} \delta n(\mathbf{q}, \omega)$. Using this relation in Eq. (22), we obtain

$$
\delta n(\mathbf{q}, \omega)=\frac{(4 \pi)^{2} e^{2}}{\epsilon_{s}} \sum_{j, L, M} \Pi_{j, L}(\omega) e^{-i(j-1) q_{x} a} j_{L}\left(q R_{j}\right) Y_{L, M}\left(\Omega_{\mathbf{q}}\right) U_{j, L M}(\omega),
$$

where $\Pi_{j, L}(\omega)$ is the density response function of the $j$-th nano shell given by an expression similar to Eq. (6) and 


$$
U_{j, L M}(\omega)=\frac{1}{\mathcal{V}} \sum_{q_{x}, \mathbf{q}_{\perp}} e^{i(j-1) q_{x} a} \frac{\delta n\left(q_{x}, \mathbf{q}_{\perp}, \omega\right)}{q_{x}^{2}+q_{\perp}^{2}} j_{L}\left(q R_{j}\right) Y_{L, M}^{*}\left(\Omega_{\mathbf{q}}\right)
$$

Substituting the expression for $\delta n(\mathbf{q}, \omega)$ given by Eq. (24) into Eq. (25), we obtain

$$
\sum_{j^{\prime}=1}^{2} \sum_{L^{\prime}=0}^{\infty} \sum_{M^{\prime}=-L^{\prime}}^{L^{\prime}}\left[\delta_{j j^{\prime}} \delta_{L L^{\prime}} \delta_{M M^{\prime}}-\Pi_{j^{\prime}, L^{\prime}}(\omega) V_{L M, L^{\prime} M^{\prime}}\left(R_{j}, R_{j^{\prime}} ; a\right)\right] U_{j^{\prime}, L^{\prime} M^{\prime}}(\omega)=0,
$$

where the Coulomb-matrix elements are

$$
\begin{aligned}
& V_{L M, L^{\prime} M^{\prime}}\left(R_{j}, R_{j^{\prime}} ; a\right)=\frac{2 e^{2}}{\pi \epsilon_{s}} \int \frac{d^{3} \mathbf{q}}{q^{2}} j_{L}\left(q R_{j}\right) j_{L^{\prime}}\left(q R_{j^{\prime}}\right) Y_{L, M}^{*}\left(\Omega_{\mathbf{q}}\right) Y_{L^{\prime}, M^{\prime}}\left(\Omega_{\mathbf{q}}\right) e^{i\left(j-j^{\prime}\right) q_{x} a} \\
= & \frac{e^{2}}{\epsilon_{s}(2 L+1) R} \delta_{L, L^{\prime}} \delta_{M, M^{\prime}} \quad \text { when } j=j^{\prime} \text { and } R_{j}=R_{j^{\prime}}=R .
\end{aligned}
$$

As a result, we obtain explicitly, by setting $j=1,2$ in turn for each of the two spheres,

$$
\begin{aligned}
& {\left[1-\frac{e^{2}}{\epsilon_{s}(2 L+1) R_{j}} \Pi_{j, L}(\omega)\right] U_{j, L M}(\omega) } \\
- & \sum_{j^{\prime} \neq j} \sum_{L^{\prime}, M^{\prime}} \Pi_{j^{\prime}, L^{\prime}}(\omega) V_{L M, L^{\prime} M^{\prime}}\left(R_{j}, R_{j^{\prime}} ; a\right) U_{j^{\prime}, L^{\prime} M^{\prime}}(\omega)=0 .
\end{aligned}
$$

If the spheres are identical, then we need only keep $j=1$, but still have to do the sum over $j^{\prime}=1,2$. The set of linear equations in (28) has nontrivial solutions provided that the determinant of the coefficient matrix for $\left\{U_{j, L M}(\omega)\right\}$ is zero. Consequently, plasmon modes with different values of $L$ on the two shells can now be coupled via the inter-sphere $\left(j \neq j^{\prime}\right)$ Coulomb interaction. Since $V_{1 L M, 2 L^{\prime} M^{\prime}}\left(R_{1}, R_{2} ; a\right) \rightarrow 0$ in the limit $a \rightarrow \infty$, the coefficient matrix becomes diagonal when $a \gg R_{1}, R_{2}$ and the plasmon-mode equation simply reduces to the result for isolated shell

$$
\prod_{L}\left[\epsilon_{1, L}(\omega)\right]^{2 L+1}\left[\epsilon_{2, L}(\omega)\right]^{2 L+1}=0
$$

where $\epsilon_{j, L}(\omega)$ is the dielectric function for the $j$-th shell. The significance of equations (28) for chosen $L, M$ is that they give explicitly the effect of the Coulomb interaction on each shell through $\epsilon_{j, L}(\omega)$ as well as the coupling between the pair of shells through the Coulomb matrix elements $V_{L M, L^{\prime} M^{\prime}}\left(R_{j}, R_{j^{\prime}} ; a\right)$, i.e., dimerization. Additionally, the nature of this coupling may be characterized in the following way when carrying out numerical calculations. For chosen $L$ and $|M| \leq L$, there are $2(2 L+1) \times 2(2 L+1)$ elements in a block sub-matrix which includes $(2 L+1)$ elements along the diagonal, equal to $\epsilon_{1, L}(\omega)$, and $(2 L+1)$ diagonal elements equal to $\epsilon_{2, L}(\omega)$. For example, if we consider the coupling between sub-matrices with angular momentum $L=1,2,3, \cdots, N$, then the dimension of the

matrix is $2 \sum_{L=1}^{N}(2 L+1)=2 N^{2}+4 N$. Specifically, if we use just the $L=1$ sub-matrix, we have a $6 \times 6$ matrix whose properties are discussed in the Appendix B

\section{NUMERICAL RESULTS}

if the radius of the buckyball is small, we may neglect the bandwidth (compared to large energy level separation) due to electron hopping between neighboring lattice sites on the sphere and simply use a spherical model of an electron gas to describe its optical response. In our numerical calculations, we chose $T=0 \mathrm{~K}$ and assume the number of occupied energy levels, $N_{F}$, is fixed. Also, the electron effective mass $\mu^{*}=0.25 m_{e}$ where $m_{e}$ is the free-electron mass. Additionally, the frequency in the polarization function is replaced by $\omega+i \gamma$ where we chose the broadening parameter $\hbar \gamma=0.05 \mathrm{eV}$. The background dielectric constant $\epsilon_{b}=2.4$ which is the same as for graphene. 

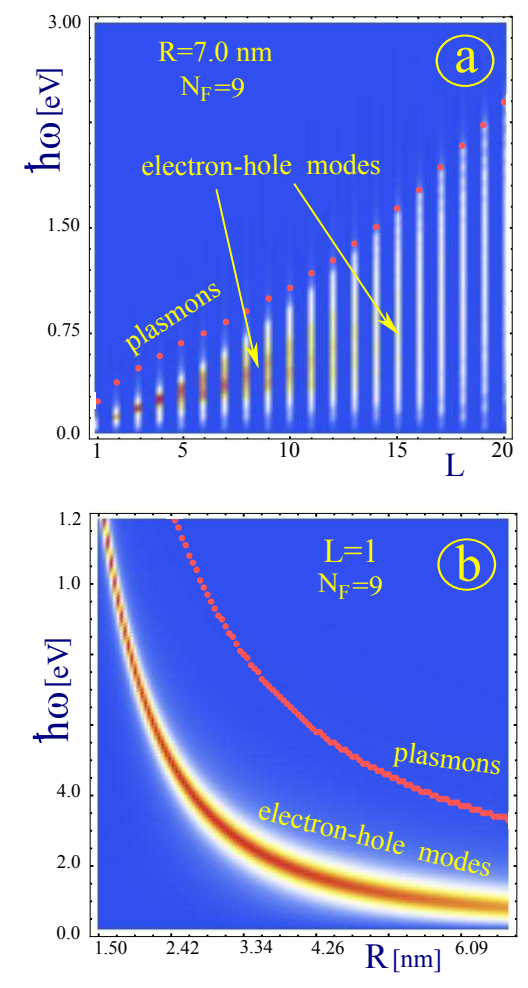

FIG. 3: (Color online) Density plots of frequencies $\omega$ for the plasmon excitations and particle-hole modes on a spherical shell as a function of $L$ (a) for $R=7 \mathrm{~nm}, N_{F}=9$ and (b) as a function of its radius $R$ for $L=1, N_{F}=9$.

Figure 3(a) shows the dependence of the plasmon excitation energy as well as that for the single-particle modes on the angular momentum quantum number $L$. Clearly, this dependence is similar to that for the 2DEG when the plasma excitation energy is plotted as a function of wave number $q$. In the long-wavelength limit, the plasmon frequency for the 2DEG has a $\sqrt{q}$ dependence which resembles the variation of frequency with $L$ in Fig.3(a) at small angular momentum. At large angular momentum, on the other hand, the plasmons are severely damped by the particle-hole modes analogous to the Landau damping of plasmon excitations in a 2DEG when $q$ is comparable with the Fermi wave vector $k_{F}$. We observe that the highest intensity (largest values of $\operatorname{Im}\left[\Pi_{L}(\omega)\right]$ ) for the electron-hole modes occur at small $L$ values, i.e., $1 \lesssim L \lesssim 3$.

The corresponding region of high plasmon intensity lies close to the upper boundary of single-particle excitations, as seen from Fig.3(a). Of course, these boundaries for electron-hole modes are determined by the chemical potential of the S2DEG, i.e., the number of electrons, as well as the electron effective mass. On the other hand, there is no analogy for the 2DEG with Fig.3(b) where we vary the radius of the S2DEG but keep the number of electrons fixed. The plasmon mode frequency decreases with increasing radius $R$ as $1 / R^{2}$ for small $R$ values but $1 / R$ for large values of $R$. After the Fermi energy $E_{F}$ has been determined, one may label the topmost occupied energy level by the angular momentum quantum number $\ell=\ell_{F}$, while the next empty level for active optical transition with $L=1$ may be labeled by $\ell^{\prime}=\ell_{F}+1$. Physically, the Fermi energy $E_{F}$ cannot be fixed for discrete energy levels due to pinning of the Fermi level, and it should be determined by the given total number of electrons, $N_{e}$, on the shell. If the total number of occupied energy levels is $N_{F}$, we find $N_{F}=\sqrt{N_{e} / 2}$ and the Fermi energy is calculated through $E_{F}=\ell_{F}\left(\ell_{F}+1\right) \hbar^{2} /\left(2 \mu^{*} R^{2}\right)$ with $\ell_{F}=N_{F}-1$. In addition, from the Fermi energy, one also gets the estimate $\left(N_{F}-1\right)^{2} \approx 6.6\left[E_{F}(\mathrm{eV})\right]\left[R^{2}(\mathrm{~nm})\right]$ for $\ell_{F} \gg 1$, implying $E_{F}$ will depend on both $N_{e}$ and $R$ at the same time.

We now turn to a description of our results in Fig. 4 for the plasmon excitations of two concentric S2DEGs when the inner radius is chosen as $R_{1}=5.0 \mathrm{~nm}$ and the outer radius $R_{2}=5.5 \mathrm{~nm}$ in (a) and $R_{2}=10.0 \mathrm{~nm}$ in (b). Since the structure is spherically symmetric, the plasma modes can still be labeled by the angular momentum quantum number $L$. Both inner and outer shells have a single-particle excitation spectrum which overlap when plotted as a function of $L$. Additionally, each S2DEG gives rise to a plasmon branch which is renormalized by the inter-shell Coulomb interaction. The two plasmon branches correspond to in-phase (symmetric) and out-of-phase (anti-symmetric) chargedensity oscillations. When the difference between the radii is small in (a), single-particle energies from the two shells almost coincide and the symmetric and anti-symmetric plasmons lie close to each other and are well above the regions 

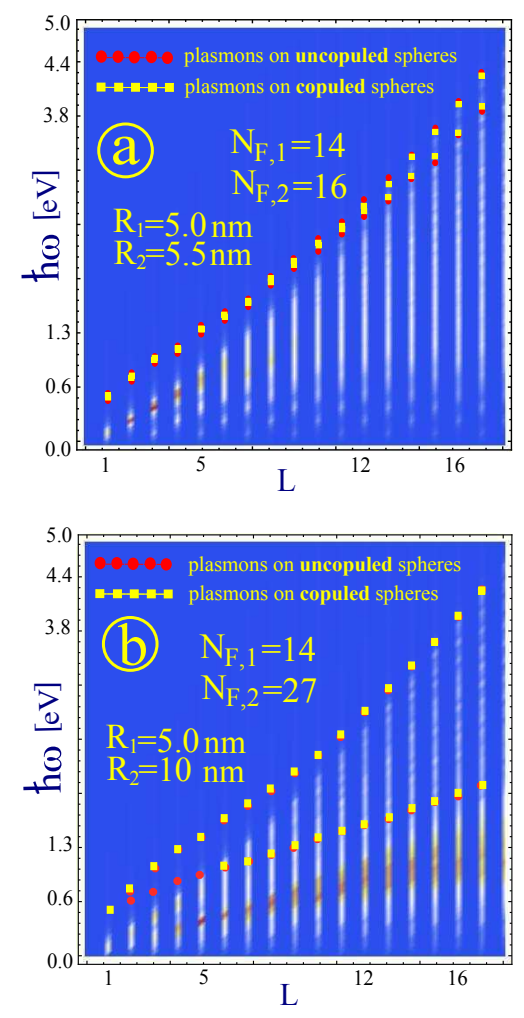

FIG. 4: (Color online) Density plot of frequency of the plasmon excitations and particle-hole modes vs. $L$ on two concentric S2DEGs for two various cases of close [in (a)] and different [in (b)] radii of inner and outer spheres. Here, we chose $N_{F}=14$ for $R_{1}=5.0 \mathrm{~nm}$ and $N_{F}=16$ for $R_{2}=5.5 \mathrm{~nm}$ in (a), while we choose $N_{F}=14$ for $R_{1}=5.0 \mathrm{~nm}$ and $N_{F}=27$ for $R_{2}=10 \mathrm{~nm}$ in (b).

where there exists Landau damping as shown in Fig.凹(a). This behavior is connected to a very weak inter-shell Coulomb interaction which is scaled by $1-\left(R_{1} / R_{2}\right)^{2 L+1}$ for $R_{1} \approx R_{2}$. As the radius of the outer S2DEG is increased, one of the plasmon frequencies is pushed down. Such an observation can be attributed to the strongly enhanced inter-shell Coulomb interaction with $R_{1} \ll R_{2}$ as $L \gg 1$. When the outer radius is much larger than the inner radius, the lower plasmon branch is strongly Landau damped by particle-hole modes and its intensity becomes very low.

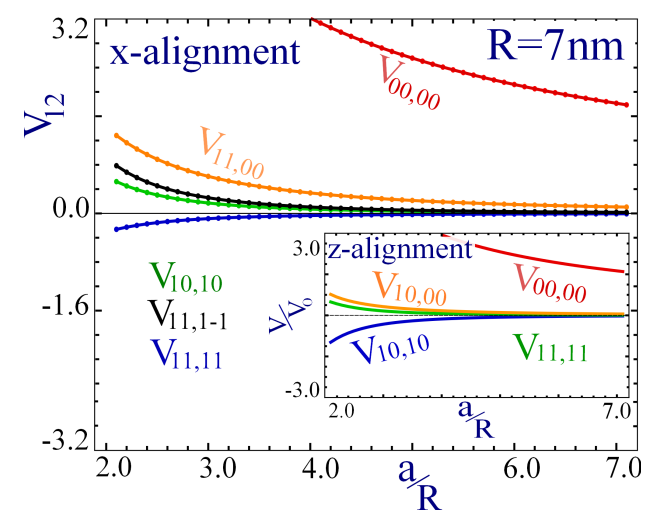

FIG. 5: (Color online) Coulomb matrix elements $\left\{V_{12}\right\}$ for two S2DEGs of radius $R_{1}=R_{2}=R$ nm in units of $V_{0}=e^{2} /\left(4 \pi \epsilon_{s} R\right)=$ $2.98 \mathrm{eV}$ for $R=7 \mathrm{~nm}$. One sphere has its center at the origin while another has its center on the $x$-axis. The separation between the centers of the two spheres is $a$ which is varied. The inset shows the dependence of the Coulomb-interaction matrix elements when two spheres have their centers along the $z$-axis for the same chosen parameters.

Additionally, we have discovered that the Coulomb interaction between the shells is not simply given by a power law but has oscillations due to the orbital motion of the S2DEG which is an interesting feature, that has not been 
discussed in the literature to our knowledge.

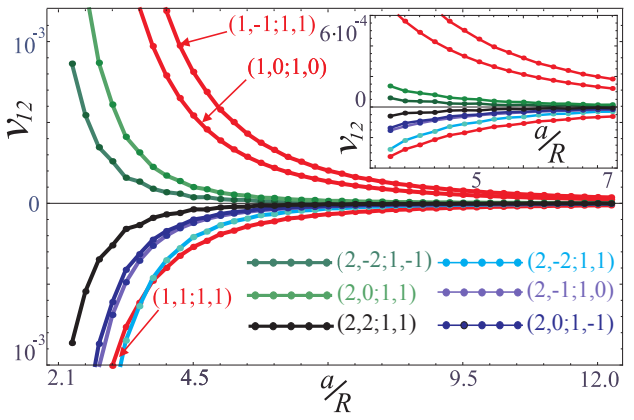

FIG. 6: (Color online) Comparison of the Coulomb matrix elements $\left\{V_{12}\right\}$ in the units $e^{2} /\left(\epsilon_{s} R\right)$ with $\epsilon_{s}=4 \pi \epsilon_{0} \epsilon_{b}$ for two S2DEGs when $L=1$ and $L=2$. Here, one sphere has its center at the origin, while the other one has its center on the $x$-axis. The radius of each S2DEG is $R_{1}=R_{2}=R=1 \mathrm{~nm}$. The separation between the centers of the spheres is $a$. The inset shows the $a$ dependence within a smaller range.

In our calculations of the plasmon frequencies for the pair of S2DEGs shown schematically in Fig.2, we must truncate the infinite matrix [22] in Eq. (28). Here, the off-diagonal matrix elements involve the Coulomb interaction $v_{1,2,3}=$ $V_{L M, L^{\prime} M^{\prime}}\left(R_{j}, R_{j^{\prime}} ; a\right)$ (see Appendix A) and their comparative values would determine whether a perturbation picture has validity. The origin of this Coulomb interaction comes from the optically-induced magnetic dipoles by a finite photon angular momentum $(\ell \neq 0)$ for anisotropic distribution of electrons on the shell. If we choose the quantization axis of angular momentum along the probe $\mathbf{E}$-field direction, the $L=0$ inter-sphere Coulomb interaction has no contribution to the system. In the presence of a finite photon angular momentum $L=1$, electron transition from the $\ell=0$ state to the $\ell=1$ state will occur. The induced magnetic dipoles associated with the $\ell=1$ states of two displaced shells will couple to each other either in phase or out of phase (split plasmon modes), leading to so-called Coulomb dimerization. This leading magnetic coupling results from the action of the magnetic field by the induced oscillating electric dipole on one sphere on the induced magnetic dipole on another sphere. Moreover, the coupling strength, which is scaled as $1 / a$ for the far-field region $\left(a \gg R, \lambda_{\mathrm{pl}}\right)$ or as $1 / a^{2}$ for the near-field range $(a \gg R$ but $\left.a \ll \lambda_{\text {pl }}\right)$, is different when two shells are displaced along the direction either parallel to the angular-momentum quantization axis (similar to $\pi$ bond for carbon atoms) or perpendicular to the axis of quantization (similar to $\sigma$ bond). Here, $a$ is the separation between two displaced spheres, $R$ is the radius of spheres, $\lambda_{\mathrm{pl}}$ is the plasmon wavelength and the coupling from acting of the magnetic field by the induced oscillating magnetic dipole on one sphere on the induced magnetic dipole on another sphere is vary small for large values of $a$. In this regard, we compare the Coulomb matrix elements in Fig. 5 when one sphere is located at the origin while the other one has its center on the $x$-axis at $(a, 0,0)$ or along the $z$-axis at $(0,0, a)$. We chose $R_{1}=R_{2}=R, L=L^{\prime}=1$ and $M, M^{\prime}=0, \pm 1$. There are fewer non-zero Coulomb matrix elements when the spheres are centered on the $z$-axis compared to when they are on the $x$-axis. Additionally, the corresponding values for these non-zero elements are not equal for the two orientations of the pair of S2DEGs, which reflects the directionality in the plasmon-plasmon spatial correlation. There are oscillations in $v_{1,2,3}$ in accordance with our semi-analytic results given for large separations $a$ in Eqs. (A8) and (A19) in Appendix A and the Coulomb interaction decreases with increasing $a$. There is only one negative Coulomb matrix element for both configurations, implying a weak bonding effect between two S2DEGs. Although the interaction $V_{00 ; 00}\left(R_{1}, R_{2} ; a\right)$ between two electric dipole moments is always positive and a dominant one due to isotropic distribution of electrons, it does not contribute to spherical plasmon excitations which require $L \geq 1$. In Fig. 6, we also compare the Coulomb matrix elements with angular momentum quantum numbers $L=1$ and $L=2$. These Coulomb matrix elements must be included if we would like to include the coupling between these two higher angular momenta. Our results in Fig. 6. however, show that to the lowest order, we may neglect these couplings since they decay fast with increasing separation $a$.

In Figs. 7 and 8 we present results of our calculations of the $L=1$ plasmon modes on a pair of coupled S2DEGs with $R_{1}=R_{2}=R$ on the $x$-axis and $z$-axis, respectively. The difference between the two plots is striking but they still have some common features. For example, the plasmon frequency is decreased as the radius of the S2DEG is increased. For a chosen radius, the plasmon frequency is slightly larger for the $z$-alignment. However, this small difference in the plasmon excitation spectrum demonstrates that the plasma-plasma interaction is spatially correlated. The intersphere Coulomb interaction lifts the degeneracy of a plasmon mode on each sphere. For $L=1$ and $M=0, \pm 1$, these two sets of modes are coupled to form three in-phase symmetric and three out-of-phase antisymmetric modes of charge-density oscillations. Therefore, one expects that the plasma mode equation would in general yield six solutions. However, some plasmon frequencies are degenerate while others may be close. We emphasize that the semi-analytic 

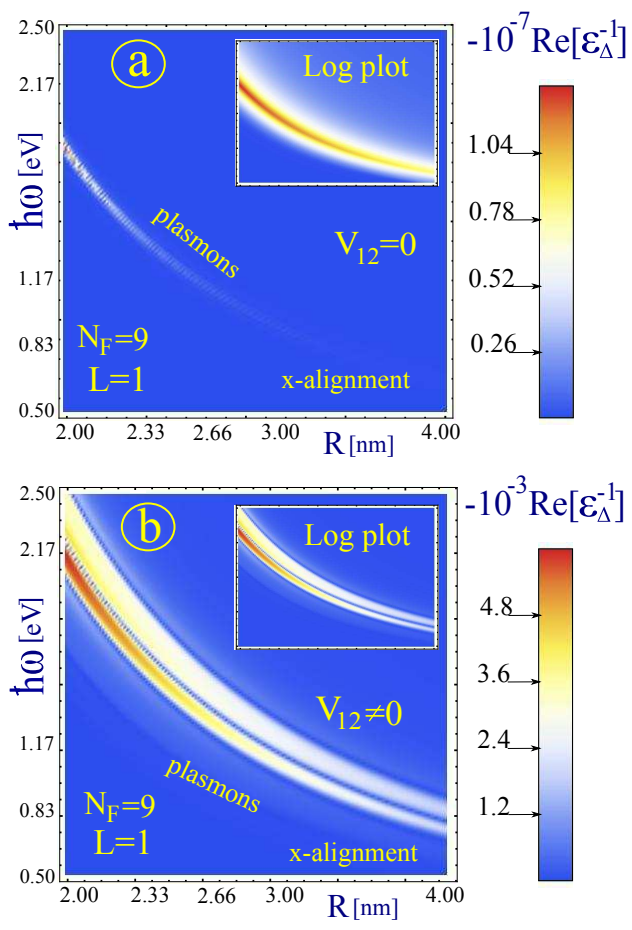

FIG. 7: (Color online) Comparison of the density plots of frequency vs. radius $R$ for plasmon excitations when $L=1$ and $N_{F}=9$ for a pair of coupled S2DEGs on the $x$-axis with the inter-sphere Coulomb interaction included $\left(V_{12} \neq 0\right)($ lower panel) or excluded $\left(V_{12}=0\right)$ (upper panel). The separation between the spheres is $a-2 R=0.1 \mathrm{~nm}$. Both insets show the logarithm of one-plus the density obtained for each pair of values of frequency and radius of the S2DEG of the corresponding results.

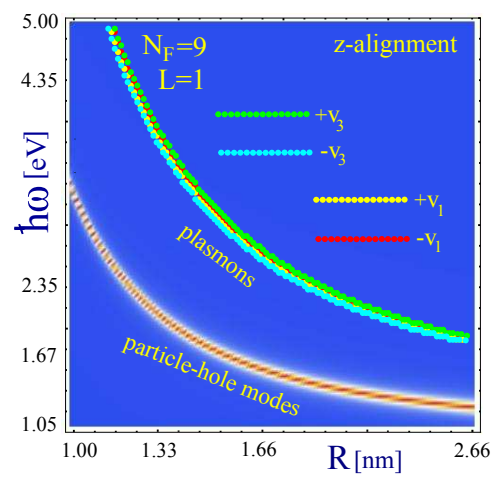

FIG. 8: (Color online) Density plots of frequency vs. radius $R$ for plasmon excitations when $L=1$ and $N_{F}=9$ for a pair of coupled S2DEGs on the $z$-axis with the inter-sphere Coulomb interaction included $\left(V_{12} \neq 0\right)$. The two different non-zero potential matrix elements are labeled $v_{1}$ and $v_{3}$ as defined in Appendix $\mathrm{A}$ The separation between the spheres is $a-2 R-0.1 \mathrm{~nm}$.

forms of the Coulomb interactions in Eqs. (A8) and (A19) do not scale as a point-like dipole-dipole coupling at large separation. The angular momentum quantum numbers $L, M$ also determine these Coulomb matrix elements, i.e., the plasmon-plasmon interaction depends on the spatial profile of an incident light beam. This implies that the bonding process in the Coulomb dimerization is directional, depending on the angular distribution of electrons for $\ell \neq 0$, similar to $\sigma$ and $\pi$ bonds between two carbon atoms. Figure 7 (b) shows that when the inter-sphere Coulomb interaction is included, the intensity of the density plots for plasmon excitations is enhanced from their values in Fig. Z(a), indicating a dimerization process between two spatially separated S2DEGs. The reason we truncated the matrix in our calculations was to see the effect of the Coulomb coupling between the shells on the lowest plasmon modes. Each of these modes has different intensity arising from the value of the loss function. By including the matrix elements which involve the $L=2$ angular momentum, there will be additional plasmon modes, which will not affect these six lowest modest substantially if the two shells are not too close to each other (see Fig.66). These results in conjunction with those in Figs. $7(\mathrm{~b})$ and 8 clearly demonstrate the existence of plasmon-coupling based dimerization, 
as well as its significance, in this system.

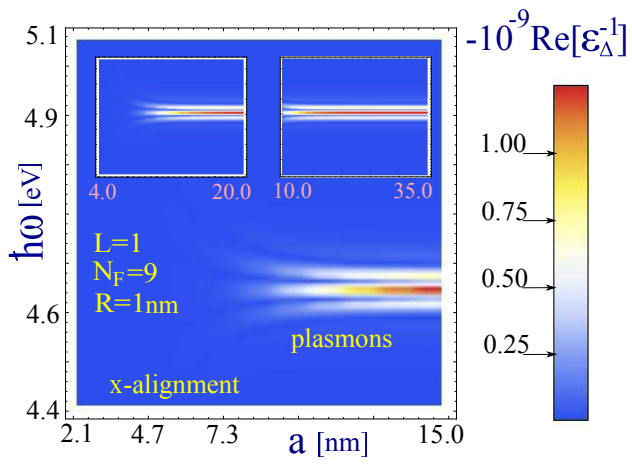

FIG. 9: (Color online) Density plot of frequency vs. separation $a$ for plasmon excitations when $L=1$ for a pair of S2DEGs each of radius $R=1 \mathrm{~nm}$. One sphere is at the origin and the other is centered on the $x$-axis. The inset shows how the plasmon excitations merge to form a single branch as $a \gg R$. Here, we have fixed $N_{F}=9$.

Finally, we investigated in Fig.9 the dependence of plasma frequency on separation $a$ between two S2DEGs with their centers on the $x$-axis. As expected, the split plasmon branches merge into a single branch for sufficiently large separations, as depicted in the inset of Fig.9. As we mentioned above, the multiplicity of plasmon modes could be less than six distinct solutions which is determined by the strengths of the inter-sphere Coulomb matrix elements. The calculated $1 / a$ dependence of the inter-sphere Coulomb interaction directly verifies the magnetic-field coupling mechanism for $a \gg R, \lambda_{\mathrm{pl}}$ in the dimerization process.

\section{CONCLUDING REMARKS}

In this paper, we presented a formalism for calculating plasma excitations for a pair of Coulomb coupled spherical electron gases. The RPA was used in this investigation. The S2DEG is a first-order approximation for calculating electronic properties of fullerenes when the lattice structure, band width from electron hopping and radial motion may be neglected. However, we may incorporate a more realistic energy band structure into the polarization function through a form factor by making use of the results presented in Ref. [18]. This would also account for the prescribed number of electrons on the fullerene. The plasmon excitation formula for a pair of Coulomb coupled S2DEGs is different when the shells are concentric compared to when these shells are side-by-side. For one thing, the angular momentum is a good quantum number for concentric shells, but all angular momenta on two displaced shells are coupled. In the latter case, we calculated the plasmon modes approximately to lowest order by including only the dominant Coulomb matrix elements which were obtained analytically. Additionally, we have demonstrated that the frequency of plasmon excitation for a pair of displaced and Coulomb coupled S2DEGs depends on both the separation between their centers as well as whether their centers lie along the axis of quantization or not. This is a consequence of the functional dependence of the Coulomb matrix elements on spatial orientation.

We note that spectral correlations have been observed experimentally for metallic nanoparticles [24] In that work, the plasmons for pairs were studied using polarization-selective total internal reflection. Their measurements show that the frequencies for the coupled plasmon modes depend on whether the incident light wave vector perpendicular and parallel to the dimer axis. Related work on dimer plasmons has been conducted by Nordlander, et al. (Ref.[25]) with the conclusion that the hybridized plasmon energy arising from individual metallic nanoparticles is determined by the orientation of the inter-particle axis with respect to the axis of polarization of the two constituents modeled as incompressible spherical liquids. Although our model differs from that in Ref. 25, 26] the conclusions about the existence of anisotropy in the plasmon excitation energies in these systems are in agreement. Similar effects are also expected to be observed in the case of nano-eggs: non-concentric multishells of nanoparticles. The hybridization of the plasmons has been proven to be an adequate and precise method to describe the plasmonic structure [27, 28]. The field enhancement, corresponding to the resonant excitation of plasmons, was reported to be much larger in the case of concentric nanoparticles, which support indirectly the concept of plasmon spatial correlation.

Generally, the angular momentum of light may be carried by either orbital motion (helicity) or spin motion (circular polarization). When the incident light has zero angular momentum with $L=0$, the electric field generated from the induced isotropic electric dipole moment for the $\ell=0$ electron state on one sphere may couple to an induced electric 
dipole moment for other $\ell=0$ electron states on another displaced sphere. However, such an isotropic plasmon excitation is associated with a change of the radial quantum number, and is prohibited in our model for spherical shells of electron gases. If a finite angular momentum of light with $L=1$ is used for incidence, on the other hand, the magnetic field generated from the induced oscillating electric dipole moment on one sphere can couple to the induced magnetic dipole moment on another displaced sphere. This unique inter-sphere magnetic (plasmon) coupling, which is associated with the magnetic dipole moment for $\ell=1$, becomes anisotropic in space, depending on the displacement of two spheres parallel or perpendicular to the direction of a probe electric field. In addition, such an oscillating electric dipole moment based inter-sphere magnetic coupling directly leads to dimerization of electron gases on two spheres. For the plasmon excitation with $L \geq 2$, the higher angular-momentum component of specific incident light beam is required, such as a helical or a Bessel light beam. In principle, the effect of plasmon coupling predicted in this paper should be experimentally observable by using light with a finite angular momentum for incidence and rotating the sample by $90^{\circ}$ for showing its directional bonding effect. The key feature presented in this paper is the broken rotational symmetry by coupling between two center-displaced S2DEGs based on photo-excited electron density fluctuations. Here, the quantization axis of the system is selected by the probe electric field. Such a directional plasmon-correlation effect will be lost if two S2DEGs are projected onto a plane, i.e., a pair of quantum rings [29], because the quantization axis is always perpendicular to the plane of the rings. For a S2DEG, we obtain a degeneracy in single-electron kinetic energies with respect to $m$ (angular momentum number along the axis of quantization). But, this degeneracy is reduced to $\pm m$ for a quantum ring. In order to completely remove the $m$-degeneracy, an external magnetic field $\mathbf{B}$ can be applied to the system. In this case, a strong magnetic field will change the S2DEG simply to a Landau quantized S2DEG [30] with kinetic energy $\sim \hbar \omega_{c}$ but change a quantum ring to a classical point mass rotating around a circle with reduced inertia and angular velocity $\omega_{c}$, where $\omega_{c}=e B / \mu^{*}$. The study of spatial correlation of magneto-S2DEGs on two displaced spheres is under investigation.

\section{Acknowledgments}

This research was supported by contract \# FA 9453-07-C-0207 of AFRL. DH would like to thank the support from the Air Force Office of Scientific Research (AFOSR).

\section{Appendix A: Calculations of Coulomb interaction matrix elements}

In Appendix $\mathrm{A}$ we demonstrate how we obtained semi-analytic expressions for the potential matrix elements $V_{L M, L^{\prime} M^{\prime}}\left(R_{1}, R_{2} ; a\right)$ with different eigenstates labeled by $L, M$ and $L^{\prime}, M^{\prime}$, corresponding to each sphere. Our primary consideration arises when the two shells are centered on the $x$-axis, i.e., an axis perpendicular to the axis of the angular momentum quantization. A simple case when the two spheres are centered on the $z$-axis will be briefly discussed at the end of Appendix $\mathrm{A}$.

\section{Plane-Wave Expansion Method}

We now describe how each of the matrix elements could be expanded as a linear combination of triple spherical Bessel function integrals. In this regard, we must evaluate the following integral

$$
\mathcal{I} \equiv \int \frac{d^{3} \mathbf{q}}{q^{2}} j_{L}\left(q R_{1}\right) j_{L^{\prime}}\left(q R_{2}\right) Y_{L, M}^{*}\left(\Omega_{\hat{\mathbf{q}}}\right) Y_{L^{\prime}, M^{\prime}}\left(\Omega_{\hat{\mathbf{q}}}\right) \mathrm{e}^{-i q_{x} a} .
$$

First, we present in spherical coordinates $\Omega_{\hat{\mathbf{q}}}=\{1, \theta, \phi\}$ and $\hat{\mathbf{e}}_{x}=\left\{1, \theta=\frac{\pi}{2}, \phi=\pi\right\}$ (Note: $\phi=$ either 0 or $\pi$ depending on whether we need to calculate $\mathrm{e}^{i q_{x} a}$ or $\left.\mathrm{e}^{-i q_{x} a}\right)$. The standard plane-wave expansion over spherical harmonics gives

$$
\mathrm{e}^{-i q_{x} a}=4 \pi \sum_{\lambda} \sum_{\mu=-\lambda}^{\lambda}(i)^{\lambda} j_{\lambda}(q a) Y_{\lambda, \mu}^{*}\left(\Omega_{\hat{\mathbf{q}}}\right) Y_{\lambda, \mu}\left(\Omega_{\hat{\mathbf{e}}_{x}}\right) .
$$

By making use of this result, the integral in Eq. (A1) turns into 


$$
\begin{gathered}
\mathcal{I}=(4 \pi) \sum_{\lambda, \mu} \int_{0}^{\infty} d q(i)^{\lambda} j_{L}\left(q R_{1}\right) j_{L^{\prime}}\left(q R_{2}\right) j_{\lambda}(q a) Y_{\lambda, \mu}\left(\Omega_{\hat{\mathbf{e}}_{x}}\right) \\
\times \int d \Omega_{\hat{\mathbf{q}}} Y_{L, M}^{*}\left(\Omega_{\hat{\mathbf{q}}}\right) Y_{L^{\prime}, M^{\prime}}\left(\Omega_{\hat{\mathbf{q}}}\right) Y_{\lambda, \mu}^{*}\left(\Omega_{\hat{\mathbf{q}}}\right) .
\end{gathered}
$$

By recalling the "triple-Y" integration formula

$$
\begin{aligned}
& \int d \Omega_{\hat{\mathbf{q}}} Y_{L, M}\left(\Omega_{\hat{\mathbf{q}}}\right) Y_{L^{\prime}, M^{\prime}}\left(\Omega_{\hat{\mathbf{q}}}\right) Y_{\lambda, \mu}\left(\Omega_{\hat{\mathbf{q}}}\right) \\
= & \sqrt{\frac{(2 L+1)\left(2 L^{\prime}+1\right)(2 \lambda+1)}{4 \pi}}\left(\begin{array}{ccc}
L & L^{\prime} & \lambda \\
0 & 0 & 0
\end{array}\right)\left(\begin{array}{ccc}
L & L^{\prime} & \lambda \\
M & M^{\prime} & \mu
\end{array}\right)
\end{aligned}
$$

and the identity $Y_{L, M}(\theta, \phi)=(-1)^{M} Y_{L,-M}^{*}(\theta, \phi)$, we finally obtain from Eq. (27)

$$
\begin{aligned}
V_{L M, L^{\prime} M^{\prime}}\left(R_{1}, R_{2} ; a\right) & =\frac{8 e^{2}}{\epsilon_{s}} \sum_{\lambda, \mu}(-1)^{\lambda / 2-M-\mu} Y_{\lambda, \mu}\left(\Omega_{\hat{\mathbf{e}}_{x}}\right) \\
& \times \sqrt{\frac{(2 L+1)\left(2 L^{\prime}+1\right)(2 \lambda+1)}{4 \pi}}\left(\begin{array}{ccc}
L & L^{\prime} & \lambda \\
0 & 0 & 0
\end{array}\right)\left(\begin{array}{ccc}
L & L^{\prime} & \lambda \\
M & M^{\prime} & \mu
\end{array}\right) \\
& \times \int_{0}^{\infty} d q j_{L}\left(q R_{1}\right) j_{L^{\prime}}\left(q R_{2}\right) j_{\lambda}(q a) .
\end{aligned}
$$

Since there are only a few non-zero terms in that summation (see next section of Appendix @), we simply write

$$
V_{L M, L^{\prime} M^{\prime}}\left(R_{1}, R_{2} ; a\right)=\sum_{\lambda} \mathcal{C}_{\lambda}\left(L, M ; L^{\prime}, M^{\prime}\right) \int_{0}^{\infty} d q j_{L}\left(q R_{1}\right) j_{L^{\prime}}\left(q R_{2}\right) j_{\lambda}(q a)
$$

For $z \rightarrow \infty$, we have

$$
j_{L}(z) \approx \frac{1}{z} \sin \left(z-\frac{\pi L}{2}\right)
$$

so that we obtain, in the limit $a \rightarrow \infty$,

$$
\begin{gathered}
V_{L M, L^{\prime} M^{\prime}}\left(R_{1}, R_{2} ; a\right) \approx \frac{1}{a} \sum_{\lambda} \mathcal{C}_{\lambda}\left(L, M ; L^{\prime}, M^{\prime}\right) \\
\quad \times \int_{0}^{\infty} \frac{d q}{q} j_{L}\left(q R_{1}\right) j_{L^{\prime}}\left(q R_{2}\right) \sin \left(q a-\frac{\pi \lambda}{2}\right),
\end{gathered}
$$

which shows that the asymptotic behavior of Coulomb interaction exhibits oscillations with respect to sphere separation $a$. 


\section{Analytic Evaluation of the Angular Integrals for Potential Matrix Elements ( $x-$ alignment)}

One may verify that it is possible to perform the angular $\phi$ and $\theta$ integrations analytically for all potential matrix elements in a relatively straightforward way. The starting point is to evaluate a three-dimensional integral in spherical coordinates:

$$
\begin{aligned}
& V_{L M, L^{\prime} M^{\prime}}\left(R_{1}, R_{2} ; a\right)=\frac{2 e^{2}}{\pi \epsilon_{s}} \int \frac{d^{3} \mathbf{q}}{q^{2}} j_{L}\left(R_{1} q\right) j_{L^{\prime}}\left(R_{2} q\right) Y_{L, M}^{*}(\theta, \phi) Y_{L^{\prime}, M^{\prime}}(\theta, \phi) \mathrm{e}^{-i q_{x} a} \\
= & \frac{2 e^{2}}{\pi \epsilon_{s}} \int_{0}^{\infty} d q j_{L}\left(R_{1} q\right) j_{L^{\prime}}\left(R_{2} q\right) \int_{0}^{\pi} \sin \theta d \theta \int_{0}^{2 \pi} d \phi Y_{L, M}^{*}(\theta, \phi) Y_{L^{\prime}, M^{\prime}}(\theta, \phi) \mathrm{e}^{-i q_{x} a} .
\end{aligned}
$$

We perform the $\phi$-integration first and label the result as $\mathcal{I}_{\phi}$. After the $\theta$-integration is completed, the final angular integral will be referred to as $\mathcal{I}_{\theta}$.

In spherical coordinates $q_{x}=q \sin \theta \cos \phi$, therefore, one writes

$$
\mathcal{I}_{\phi}(q, \theta)=\int_{0}^{2 \pi} d \phi \mathrm{e}^{-i a q \sin \theta \cos \phi} .
$$

In order to obtain a closed-form analytic result from Eq. (A10), we use the Jacobi-Anger identity

$$
\mathrm{e}^{i \xi \cos \phi}=\sum_{m=-\infty}^{\infty} i^{m} J_{m}(\xi) \mathrm{e}^{i m \phi},
$$

where $J_{m}(x)$ stands for Bessel functions of the first kind.

It follows from Eq. (A11) and the exponential $\phi$-dependence of spherical harmonics $Y_{L, \pm M}(\theta, \phi)$, that the order of the only remaining non-zero term contains the Bessel function, determined by the difference between $M$ and $M^{\prime}$ values, namely by $\left|M-M^{\prime}\right|$. Consequently, we classify all the results of $\phi$-integration by $\left|M-M^{\prime}\right|$. For our present consideration with $L, L^{\prime}=1$ this difference could only be 0,1 or 2 .

Since the only way to obtain an imaginary result for $\mathcal{I}_{\phi}(q, \theta)$ in Eq. A2 comes from $i^{m}$ (see Eq. A17), we see that

$$
\mathcal{I}_{\phi}(q, \theta) \backsim i^{\left|M-M^{\prime}\right|} .
$$

Therefore, the result of $\phi$-integration (and, consequently, the potential matrix element) will be imaginary if $\mid M-$ $M^{\prime} \mid=1$. For the relevant case $L=L^{\prime}=1$, all the elements with $\left|M-M^{\prime}\right|=1$, i.e., $M= \pm 1, M^{\prime}=0$, or vice versa, are equal to zero due to a specific symmetry in the $\theta$-integration (check the matrix elements in Eq. (B6)), so that all the potential matrix elements are real.

\section{Summary of Relevant Potential Matrix Elements for $L=1$}

As we are now going to obtain, the modifications $\mathrm{e}^{-i q a \sin \theta \cos \phi} \Rightarrow \mathrm{e}^{i q \sin \theta \cos \phi}, \mathrm{e}^{i \phi} \Rightarrow \mathrm{e}^{-i \phi}$ and $\mathrm{e}^{2 i \phi} \Rightarrow \mathrm{e}^{-2 i \phi}$ do not alter the values of all real $\phi$-integrals. As long as only these elements result in a non-zero $\theta$-integral, the potential sub-matrices $A$ and $B$ in Eq. (B1) are identical.

We noted in Sec.A2 that the result of the $\phi$-integration is determined by $\left|M-M^{\prime}\right|$ and does not depend on each individual $M, M^{\prime}$ value. Consequently, there are only three different non-zero potential matrix elements, which will be later referred to as $v_{1,2,3}$, respectively.

Let us now briefly provide the integration results for each non-zero potential matrix element $V_{L M, L^{\prime} M^{\prime}}\left(R_{1}, R_{2} ; a\right)$. As mentioned above, $L=L^{\prime}=1$ for all cases. 
a. $\quad M=M^{\prime}=0$

$$
\begin{aligned}
& \mathcal{I}_{\phi}(q, \theta)=\int_{0}^{2 \pi} d \phi \mathrm{e}^{-i q a \sin \theta \cos \phi}\left|Y_{1,0}(\theta, \phi)\right|^{2}=\frac{3}{2} \cos ^{2} \theta J_{0}(a q \sin \theta), \\
& \mathcal{I}_{\theta}(q)=\frac{3}{2} \int_{0}^{\pi} J_{0}(a q \sin \theta) \sin \theta \cos ^{2} \theta d \theta=3 \frac{\sin (a q)-a q \cos (a q)}{(a q)^{3}}, \\
& v_{3} \equiv V_{10,10}\left(R_{1}, R_{2} ; a\right)=\frac{6 e^{2}}{\pi \epsilon_{s}} \int_{0}^{\infty} d q \frac{\sin (a q)-a q \cos (a q)}{(a q)^{3}} j_{1}\left(R_{1} q\right) j_{1}\left(R_{2} q\right) .
\end{aligned}
$$

Here we write the final answer, taking into account the coefficient $2 e^{2} / \pi \epsilon_{s}$ for all potential matrix elements.

$$
\text { b. } \quad M=M^{\prime} \neq 0
$$

$$
\begin{aligned}
& \mathcal{I}_{\phi}(q, \theta)=\int_{0}^{2 \pi} \mathrm{e}^{-i q a \sin \theta \cos \phi}\left|Y_{1, \pm 1}(\theta, \phi)\right|^{2} d \phi=\frac{3}{4} \sin ^{2} \theta J_{0}(a q \sin \theta), \\
& \mathcal{I}_{\theta}(q)=\frac{3}{4} \int_{0}^{\pi} J_{0}(a q \sin \theta) \sin ^{3} \theta d \theta=\frac{3}{2} \frac{a q \cos (a q)-\left((a q)^{2}-1\right) \sin (a q)}{(a q)^{3}}, \\
& v_{1} \equiv V_{1-1,1-1}\left(R_{1}, R_{2} ; a\right)=V_{11,11}\left(R_{1}, R_{2} ; a\right)=\frac{3 e^{2}}{\pi \epsilon_{s}} \int_{0}^{\infty} d q \frac{a q \cos (a q)+\left((a q)^{2}-1\right) \sin (a q)}{(a q)^{3}} j_{1}\left(R_{1} q\right) j_{1}\left(R_{2} q\right) .
\end{aligned}
$$

$$
\text { c. }\left|M-M^{\prime}\right|=1 \Rightarrow M \vee M^{\prime}=0
$$

$$
\begin{aligned}
& \mathcal{I}_{\phi}(q, \theta) \simeq \int_{0}^{2 \pi} d \phi \mathrm{e}^{-i q a \sin \theta \cos \phi} \mathrm{e}^{ \pm i \phi}=2 \pi i J_{1}(a q \sin \theta), \\
& \mathcal{I}_{\theta}(q) \simeq \int_{0}^{\pi} J_{1}(a q \sin \theta) \sin ^{2} \theta \cos \theta d \theta=0 .
\end{aligned}
$$

Therefore, we confirm our previous finding, that all potential matrix elements with one $M= \pm 1, M^{\prime}=0$ and vice versa are equal to zero (four elements in each sub-matrix). 
d. $\left|M-M^{\prime}\right|=2 \Rightarrow M=-M^{\prime}$

$$
\begin{aligned}
& \mathcal{I}_{\phi}(q, \theta)=\int_{0}^{2 \pi} d \phi\left[Y_{1, \pm 1}(\theta, \phi)\right]^{*} Y_{1, \mp 1}(\theta, \phi) \mathrm{e}^{-i q a \sin \theta \cos \phi}=\frac{3}{4} \sin \theta^{2} J_{2}(a q \sin \theta) \\
& \mathcal{I}_{\theta}(q)=\frac{3}{4} \int_{0}^{\pi} J_{2}(a q \sin \theta) \sin ^{3} \theta d \theta=-\frac{3}{2} \frac{3 a q \cos (a q)-\left((a q)^{2}-1\right) \sin (a q)}{(a q)^{3}} \\
& v_{2} \equiv V_{1-1,11}\left(R_{1}, R_{2} ; a\right)=V_{11,1-1}\left(R_{1}, R_{2} ; a\right)=-\frac{3 e^{2}}{\pi \epsilon_{s}} \int_{0}^{\infty} d q \frac{3 a q \cos (a q)-\left((a q)^{2}-3\right) \sin (a q)}{(a q)^{3}} j_{1}\left(R_{1} q\right) j_{1}\left(R_{2} q\right) .
\end{aligned}
$$

\section{4. z-alignment}

We now turn to a brief discussion of the case when the two spheres have their centers on the $z$-axis, which is also the axis of the angular momentum quantization. Significant simplification comes from the fact that the exponential term $\mathrm{e}^{-i q_{z} a}=\mathrm{e}^{-i q a \cos \theta}$ which does not depend on the azimuthal angle $\phi$. Consequently, the $\phi$-dependence of each potential matrix element is now determined solely by the exponential part or phase of the spherical harmonics. Since

$$
\int_{0}^{2 \pi} d \phi \mathrm{e}^{i\left(M-M^{\prime}\right) \phi}=2 \pi \delta_{M M^{\prime}}
$$

we can conclude that

(a) only elements with $M=M^{\prime}$ are non-zero;

(b) $V_{1-1,1-1}\left(R_{1}, R_{2} ; a\right)=V_{11,11}\left(R_{1}, R_{2} ; a\right)$.

Consequently, we need to evaluate only two non-zero elements, contributing to the plasmon equations, namely $V_{1-1,1-1}\left(R_{1}, R_{2} ; a\right)=V_{11,11}\left(R_{1}, R_{2} ; a\right)$ with $M=M^{\prime} \neq 0$ as well $V_{10,10}\left(R_{1}, R_{2} ; a\right)$. The calculation is now straightforward:

$$
\begin{aligned}
& v_{1} \equiv V_{11,11}\left(R_{1}, R_{2} ; a\right)=\int_{0}^{\infty} d q j_{1}\left(R_{1} q\right) j_{1}\left(R_{2} q\right) \int_{0}^{\pi} \mathrm{e}^{-i q a \cos \theta} \sin \theta d \theta \int_{0}^{2 \pi} d \phi\left|Y_{1,1}(\theta ; \phi)\right|^{2}= \\
& =\frac{6 e^{2}}{\pi \epsilon_{s}} \int_{0}^{\infty} d q \frac{\sin (a q)-a q \cos (a q)}{(a q)^{3}} j_{1}\left(R_{1} q\right) j_{1}\left(R_{2} q\right) .
\end{aligned}
$$

Here, again, we provide the final answer, taking into account the coefficient $2 e^{2} / \pi \epsilon_{s}$ for all potential matrix elements.

Correspondingly, the remaining potential $V_{10,10}\left(R_{1}, R_{2} ; a\right)$ is as follows:

$$
\begin{aligned}
& v_{3} \equiv V_{10,10}\left(R_{1}, R_{2} ; a\right)=\int_{0}^{\infty} d q j_{1}\left(R_{1} q\right) j_{1}\left(R_{2} q\right) \int_{0}^{\pi} d \theta \mathrm{e}^{-i q a \cos \theta} \sin \theta \int_{0}^{2 \pi} d \phi\left(Y_{1,0}(\theta ; \phi)\right)^{2} \\
& =\frac{6 e^{2}}{\pi \epsilon_{s}} \int_{0}^{\infty} d q \frac{\left((a q)^{2}-2\right) \sin (a q)+2 a q \cos (a q)}{(a q)^{3}} j_{1}\left(R_{1} q\right) j_{1}\left(R_{2} q\right) .
\end{aligned}
$$

We assign $v_{3}$ to the second non-zero potential matrix element to keep the notations uniform with the previous section. 
Finalizing this Appendix section, we provide the expression for $V_{00,00}\left(R_{1}, R_{2} ; a\right)$, representing the highest values of the interaction potential. This element, however, does not provide any contribution to the plasmon equations, since the polarization function is zero for $\mathrm{L}=0$ :

$$
V_{00,00}\left(R_{1}, R_{2} ; a\right)=\frac{3 e^{2}}{\pi \epsilon_{s}} \int_{0}^{\infty} d q j_{0}(a q) j_{0}\left(R_{1} q\right) j_{0}\left(R_{2} q\right) .
$$

One can easily verify exactly that the same expression could be obtained for $V_{00,00}\left(R_{1}, R_{2} ; a\right)$ in the case of two $x$-aligned spherical shells, which could be explained by the fact the interaction between the shells with $L=0=L^{\prime}=0$ is obviously spherically-symmetric.

\section{Appendix B: Matrix Transformations and the Calculations of Determinants}

\section{1. $x$-aligned shells}

For us to simplify the evaluation of the determinant, we use a property of the determinant of a block matrix, i.e., for such a matrix

$$
\underline{\mathcal{M}}_{B}=\left[\begin{array}{ll}
\underline{\mathcal{D}}_{1} & \underline{\mathcal{A}} \\
\underline{\underline{\mathcal{B}}} & \underline{\underline{D}}_{2}
\end{array}\right]
$$

its determinant is given by $\operatorname{Det}\left[\underline{\mathcal{M}}_{B}\right]=\operatorname{Det}\left[\underline{\mathcal{D}}_{1} \otimes \underline{\mathcal{D}}_{2}-\underline{\mathcal{A}} \otimes \underline{\mathcal{B}}\right.$. For all separations $a>R_{1}+R_{2}$, the Coulomb matrix elements in the diagonal blocks $\underline{\mathcal{D}}_{1}$ and $\underline{\mathcal{D}}_{2}$ are larger than those in the off-diagonal blocks $\underline{\mathcal{A}}$ and $\underline{\mathcal{B}}$.

We now write explicitly

$$
\underline{\mathcal{D}}_{1}=\left[\begin{array}{ccc}
\epsilon_{L=1}\left(R_{1}, \omega\right) & 0 & 0 \\
0 & \epsilon_{L=1}\left(R_{1}, \omega\right) & 0 \\
0 & 0 & \epsilon_{L=1}\left(R_{1}, \omega\right)
\end{array}\right],
$$

and

$$
\underline{\mathcal{D}}_{2}=\left[\begin{array}{ccc}
\epsilon_{L=1}\left(R_{2}, \omega\right) & 0 & 0 \\
0 & \epsilon_{L=1}\left(R_{2}, \omega\right) & 0 \\
0 & 0 & \epsilon_{L=1}\left(R_{2}, \omega\right)
\end{array}\right] .
$$

Let us consider identical spheres, i.e., equal radius $R_{1}=R_{2}=R$ and chemical potential $\mu_{1}=\mu_{2}$. In such a case, we have

$$
\operatorname{Det}\left[\mathcal{D}_{1}\right]=\operatorname{Det}\left[\mathcal{D}_{2}\right]=\left[\epsilon_{L=1}(R, \omega)\right]^{3}=\left[1-\frac{e^{2}}{3 \epsilon_{s} R} \Pi_{L=1}(\omega)\right]^{3},
$$

and, correspondingly, since both blocks $\mathcal{D}_{1}$ and $\mathcal{D}_{2}$ are diagonal, we obtain

$$
\operatorname{Det}\left[\underline{\mathcal{D}}_{1} \otimes \underline{\mathcal{D}}_{2}\right]=\left[\epsilon_{L=1}(R, \omega)\right]^{6}=\left[1-\frac{e^{2}}{3 \epsilon_{s} R} \Pi_{L=1}(\omega)\right]^{6} .
$$

From these results, we see that a density plot of the imaginary part of $1 / \operatorname{Det}\left[\underline{\mathcal{M}}_{B}\right]$ will show that the frequencies of the particle-hole excitations and the plasmons will appear almost as a power-law dependence on the imaginary part of $1 /\left[\epsilon_{L=1}(R, \omega)\right]^{6}$, with a correction due to the inter-sphere Coulomb interaction.

Previously, we obtained the potential matrices in Eq. (28) with given $v_{1,2}$ by 


$$
\underline{\mathcal{A}}=\underline{\mathcal{B}}=\Pi_{L=1}(R, \omega)\left[\begin{array}{ccc}
v_{1} & 0 & v_{2} \\
0 & v_{3} & 0 \\
v_{2} & 0 & v_{1}
\end{array}\right] .
$$

Therefore, $\operatorname{Det}[\underline{\mathcal{A}}]=\operatorname{Det}[\underline{\mathcal{B}}]=\left(v_{1}-v_{2}\right)\left(v_{1}+v_{2}\right) v_{3}$ and their product $\underline{\mathcal{A}} \otimes \underline{\mathcal{B}}$ for $R_{1}=R_{2}=R$ yields the off-diagonal corrections in the following form

$$
\underline{\mathcal{A}} \otimes \underline{\mathcal{B}}=\left[\Pi_{L=1}(R, \omega)\right]^{2}\left[\begin{array}{ccc}
\left(v_{1}\right)^{2}+\left(v_{2}\right)^{2} & 0 & 2 v_{1} v_{2} \\
0 & \left(v_{3}\right)^{2} & 0 \\
2 v_{1} v_{2} & 0 & \left(v_{1}\right)^{2}+\left(v_{2}\right)^{2}
\end{array}\right] .
$$

Finally, the complete determinant, which yields the electronic excitations, can expressed as

$$
\operatorname{Det}\left[\underline{\mathcal{D}}_{1} \otimes \underline{\mathcal{D}}_{2}-\underline{\mathcal{A}} \otimes \underline{\mathcal{B}}\right]=\operatorname{Det}\left[\begin{array}{ccc}
d_{1} & 0 & -2 \mathcal{Q}_{1} v_{1} v_{2} \\
0 & d_{2} & 0 \\
-2 \mathcal{Q}_{1} v_{1} v_{2} & 0 & d_{2}
\end{array}\right],
$$

where

$$
\begin{aligned}
d_{1} & =\epsilon_{L=1}\left(R_{1}, \omega\right) \epsilon_{L=1}\left(R_{2}, \omega\right)-\mathcal{Q}_{1}\left[\left(v_{1}\right)^{2}+\left(v_{2}\right)^{2}\right], \\
d_{2} & =\epsilon_{L=1}\left(R_{1}, \omega\right) \epsilon_{L=1}\left(R_{2}, \omega\right)-\mathcal{Q}_{1}\left(v_{3}\right)^{2}, \\
\mathcal{Q}_{1} & =\frac{2 e^{2}}{\pi \epsilon_{s}} \Pi_{L=1}\left(R_{1}, \omega\right) \Pi_{L=1}\left(R_{2}, \omega\right) .
\end{aligned}
$$

\section{2. $z$-aligned shells}

From the previous discussion it follows that, for the $q_{z}$-case, each potential sub-matrix $\underline{\mathcal{A}}$ and $\underline{\mathcal{B}}$ is diagonal and has the following form:

$$
\underline{\mathcal{A}}=\underline{\mathcal{B}}=\left[\begin{array}{ccc}
v_{1} & 0 & 0 \\
0 & v_{3} & 0 \\
0 & 0 & v_{1}
\end{array}\right]
$$

with $v_{1}$ and $v_{3}$ given by Eqs. (A18) and (A19). The reduced $3 \times 3$ matrix is

$$
\left[\begin{array}{ccc}
\epsilon_{L=1}\left(R_{1}, \omega\right) \epsilon_{L=1}\left(R_{2}, \omega\right)-\mathcal{Q}_{2} v_{1}^{2} & 0 & 0 \\
0 & \epsilon_{L=1}\left(R_{1}, \omega\right) \epsilon_{L=1}\left(R_{2}, \omega\right)-\mathcal{Q}_{2} v_{3}^{2} & 0 \\
0 & 0 & \epsilon_{L=1}\left(R_{1}, \omega\right) \epsilon_{L=1}\left(R_{2}, \omega\right)-\mathcal{Q}_{2} v_{1}^{2}
\end{array}\right],
$$

where $\mathcal{Q}_{2}=\Pi_{L=1}\left(R_{1}, \omega\right) \Pi_{L=1}\left(R_{2}, \omega\right)$ and $\Pi_{L}\left(R_{j}, \omega\right)$ is the polarization function for the shell of radius $R_{j}$ given in Eq. (6). This leads to the following equations for plasmon modes

$$
\begin{aligned}
& \epsilon_{L=1}\left(R_{1}, \omega\right) \epsilon_{L=1}\left(R_{2}, \omega\right) \pm\left[\Pi_{L=1}\left(R_{1}, \omega\right) \Pi_{L=1}\left(R_{2}, \omega\right)\right]^{1 / 2} v_{1}=0 \\
& \epsilon_{L=1}\left(R_{1}, \omega\right) \epsilon_{L=1}\left(R_{2}, \omega\right) \pm\left[\Pi_{L=1}\left(R_{1}, \omega\right) \Pi_{L=1}\left(R_{2}, \omega\right)\right]^{1 / 2} v_{3}=0
\end{aligned}
$$


[1] T. Inaoka, Surf. Sci. 273, 191 (1992).

[2] J. Tempere, I. F. Silvera and J. T. Devreese, Phys. Rev. B 65, 195418 (2002).

[3] C. Yannouleas, E. N. Bogachek and U. Landman, Phys. Rev. B 53, 10225 (1996).

[4] M. F. Lin and K. W.-K. Shung, Phys. Rev. B 47, 6617 (1993).

[5] G. Gumbs and G. R. Aizin, Phys. Rev. B 65, 195407 (2002).

[6] D. Östling, P. Apell and A. Rosen, Europhys. Lett. 21, 539 (1993).

[7] G. Gensterblum, J. J. Pireaux, R. Gaudano, J. P. Vigneron, A. A. Lucas and W. Krätschmer, Phys. Rev. Lett. 67, 2171 (1991).

[8] E. Schmen, J. Fink and W. Krätschmer, Europhys. Lett., 17, 51 (1992).

[9] E. Osawa, Kagaku (Kyoto) 25, 854 (1970) [in Japanese].

[10] J. F. Anacleto and M. A. Quilliam, Anal. Chem. 65, 2236 (1993).

[11] H. W. Kroto, J. R. Heath, S. C. O'Brien, R. F. Curl and R. E. Smalley, Nat. 318, 162 (1985).

[12] S. Iijima, J. Crystal Growth 50, 675 (1980).

[13] P. R. Buseck, S. J. Tsipursky and R. Hettich, Sci. 257, 215 (1992).

[14] J. Cami, J. Bernard-Salas, E. Peeters and S. E.Malek, Sci. 329, 180 (2010).

[15] C. A. Poland, R. Duffin, I. Kinloch, A. Maynard, W. A. H. Wallace, A. Seaton, V. Stone, S. Brown, W. MacNee and K. Donaldson, Nat. Nanotechn. 3, 423 (2008).

[16] P. Longe, Solid State Commun. 97, 857 (1996).

[17] V. K. Voora, L. S. Cederbaum and K. D. Jordan, J. Phys. Chem. Lett. 4, 6 (2013).

[18] A. K. Belyaev, A. S. Tiukanov, A. I. Toropkin, V. K. Ivanov, R. G. Polozkov and A. V. Solov'yov, Physica Scripta, 80, 048121 (2009).

[19] M. T. Michalewicz and M. P. Das, Solid State Commun. 84, 1121 (1992).

[20] G. Gumbs and G. R. Aizin, Phys. Rev. B 65, 195407 (2002).

[21] M. F. Lin and K. W.-K. Shung, Phys. Rev. B 48, 5567 (1993).

[22] V. V. Rotkin and R. A. Suris, Solid State Commun. 97, 183 (1006).

[23] Godfrey Gumbs, Antonios Balassis, Andrii Iurov, and Paula Fekete, The Scientific World Journal, vol. 2014, 726303 (2014).

[24] Yang, Shu-Chun and Kobori, Hiromu and He, Chieh-Lun and Lin, Meng-Hsien and Chen, Hung-Ying and Li, Cuncheng and Kanehara, Masayuki and Teranishi, Toshiharu and Gwo, Shangjr, Nano Letters, 10, 2 (2010).

[25] Nordlander, P. and Oubre, C. and Prodan, E. and Li, K. and Stockman, M. I., Nano Letters, 4, 5 (2004).

[26] Prodan, E. and Radloff, C. and Halas, N. J. and Nordlander, P., Science, 302, 5644 (2003).

[27] Y.Wu and P.Nordlander, The Journal of Chemical Physics, 125, 125, 124708 (2006).

[28] Bardhan, Rizia and Mukherjee, Shaunak and Mirin, Nikolay A. and Levit, Stephen D. and Nordlander, Peter and Halas, Naomi J., The Journal of Physical Chemistry C, 114, 16 (2010).

[29] D. H. Huang and G. Gumbs, Phys. Rev. B 46, 4147 (1992).

[30] D. H. Huang and G. Gumbs, Phys. Lett. A 167, 396 (1992). 